\title{
Control of spatial quantum fluctuations using photonic crystals
}

\author{
María Moreno, Damiá Gomila and Roberta Zambrini \\ Instituto de Física Interdisciplinar y de Sistemas Complejos (CSIC-UIB), Campus Universitat \\ Illes Balears, 07122 Palma de Mallorca, Spain
}

\begin{abstract}
Optical parametric oscillators emit light with non-classical correlations between opposite spatial modes (twin beams). We consider these devices in presence of an intracavity photonic crystal, modeled by a spatial modulation of the refractive index. The introduction of photonic crystals allows to control not only the macroscopic transverse profile of the emitted light beam but also its quantum fluctuations. We employ the Q representation to study pump and signal spatial correlations.
\end{abstract}

Keywords: Quantum fluctuations, quantum noise, quantum images, optical spatio-temporal dynamics, and photonic crystals

\section{INTRODUCTION}

Interaction between light and matter allows to transform coherent laser states in nonclassical ones. In particular, non linear optical cavities are known to generate intense non-classical light beams, showing squeezing and entanglement. ${ }^{1}$ In some cases quantum correlations appear not only in the whole beam but also when a portion of it or a mode in a certain basis is detected. ${ }^{2}$ Examples include quantum correlations between Fourier or orbital angular momentum modes with applications in parallel communications and imaging. ${ }^{3}$

Due to non linearity and cavity losses these devices have also been studied in the context of self-organization. Indeed, for large Fresnel numbers the emitted beams can exhibit not only temporal instability but also spontaneous pattern formation. These phenomena are often associated to quantum effects, as predicted, for instance, in light patterns emitted by parametric oscillators ${ }^{4-8}$ and Kerr cavities. ${ }^{9,10}$

In this work we consider a prototype non linear cavity modified by the presence of an intracavity photonic crystal, inducing a periodic modulation of the refractive index in the transverse plane. Previous analysis of similar models have shown the possibility to inhibit the phenomenon of pattern formation by use of photonic crystals. ${ }^{11,12}$ In other words, the instability threshold moves to larger values of the pump. We will show the role and the importance of photonic crystals in a type I degenerate optical parametric oscillator (DOPO) in order to control not only the spatial instability and the macroscopic transverse profile of the emitted light but also to engineer the distribution of the spatial quantum fluctuations.

This paper is organized as follows: in Sect. 2 we introduce the model based on the use of the Husimi Q representation. The effect of the photonic crystal on the instability threshold is discussed in Sect. 3. Quantum fluctuations and correlations of the DOPO are analyzed in Sect. 4.

\section{THE MODEL: TYPE I DOPO}

The intracavity dynamics in a DOPO is described by the boson spatial modes $\hat{A}_{0}(\vec{x}, t)$ and $\hat{A}_{1}(\vec{x}, t)$, respectively at the pump frequency $2 \omega$, and signal frequency $\omega$, with standard equal-time commutation relations ${ }^{4}$

$$
\left[\hat{A}_{i}(\vec{x}, t), \hat{A}_{j}^{\dagger}\left(\vec{x}^{\prime}, t\right)\right]=\delta_{i j} \delta\left(\vec{x}-\vec{x}^{\prime}\right), \quad i, j=0,1
$$

Further author information:

Send correspondence to R.Z.: E-mail: roberta@ifisc.uib-csic.es, Telephone: +34971259519

Quantum Communications and Quantum Imaging VI, edited by Ronald E. Meyers, Yanhua Shih, Keith S. Deacon, Proc. of SPIE Vol. 7092, 709205, (2008) · 0277-786X/08/\$18 - doi: 10.1117/12.795998 
where $\vec{x}$ denotes the transverse coordinate(s). The intracavity fields constitute an open device modeled within a statistical approach in the Schrödinger picture by a Master equation for the reduced density operator $\hat{\rho}:^{13,14}$

$$
\frac{\partial \hat{\rho}}{\partial t}=\frac{1}{i \hbar}[\hat{H}, \hat{\rho}]+\hat{\Lambda} \hat{\rho} .
$$

We consider a plane one-sided cavity, hence the Liouvillian accounting for dissipation through the partially reflecting mirror is given by

$$
\hat{\Lambda} \hat{\rho}=\sum_{i=0,1} \gamma_{i} \int d^{2} \vec{x}\left\{\left[\hat{A}_{i}(\vec{x}), \hat{\rho} \hat{A}_{i}^{\dagger}(\vec{x})\right]+\left[\hat{A}_{i}(\vec{x}) \hat{\rho}, \hat{A}_{i}^{\dagger}(\vec{x})\right]\right\} .
$$

The Hamiltonian operator is the sum of three terms:

$$
\hat{H}_{0}=\hbar \int d^{2} \vec{x} \sum_{i=0,1}\left[\gamma_{i} \hat{A}_{i}^{\dagger}(\vec{x})\left(\Delta_{i}(\vec{x})-a_{i} \nabla^{2}\right) \hat{A}_{i}(\vec{x})\right]
$$

describing free propagation of fields in the cavity within the mean field approximation,

$$
\hat{H}_{e x t}=i \hbar \int d^{2} \vec{x} E\left[\hat{A}_{0}^{\dagger}(\vec{x})-\hat{A}_{0}(\vec{x})\right]
$$

accounting for interaction with the external pump $E$ and

$$
\hat{H}_{i n t}=i \hbar \frac{g}{2} \int d^{2} \vec{x}\left[\hat{A}_{0}(\vec{x}) \hat{A}_{1}^{\dagger}(\vec{x})-\hat{A}_{0}^{\dagger}(\vec{x}) \hat{A}_{1}^{2}(\vec{x})\right]
$$

due to the non linear interaction between first and second harmonic. The intracavity photonic crystal is modeled by spatial dependent detunings for the pump $\Delta_{0}(\vec{x})$ and for the signal $\Delta_{1}(\vec{x})$. Here we will consider sinusoidal modulations.

A standard procedure allows to map density operators in state space into quasi-probability distribution densities on phase space that can be used to calculate ensemble averages of operators in defined orderings. ${ }^{15,16}$ In particular, Eq. (2) is converted into an equation of motion for a quasi-probability distribution in the phasespace of radiation fields $\alpha_{i}(\vec{x})$, associated with the operators $\hat{A}_{i}(\vec{x})$. In general, the presence of non linearities leads to a functional differential equation for the quasi-probability that is not of the Fokker-Planck type. A short overview is reported in a previous paper ${ }^{8}$ where an approximated method based in the Q representation is introduced. The Q representation corresponds to anti-normal ordering of field operators and corresponds to simultaneous measurements of orthogonal quadratures, as limited by the Heisenberg principle, in a eight-port homodyne detector. ${ }^{17}$ The most important property of this representation is that it satisfies the requirements for a true probability distribution. In fact the Q-representation may be defined as the diagonal matrix elements of the density operator in the space of coherent states

$$
Q\left(\alpha_{0}, \alpha_{1}\right)=\frac{1}{\pi}<\alpha_{0}, \alpha_{1}|\hat{\rho}| \alpha_{0}, \alpha_{1}>
$$

and so is both positive and bounded. ${ }^{16}$ In spite of the positivity and regularity of the $\mathrm{Q}$ representation, its dynamical equation for optical parametric oscillators suffers of negative diffusion if

$$
\left|\alpha_{0}(\vec{x}, t)\right|>\frac{2 \gamma_{1}}{g} .
$$

The possibility to obtain a positive solution in presence of a negative diffusion ${ }^{18}$ lies in the presence of a restricted ensemble of initial conditions. ${ }^{8}$ A Q representation with a doubled phase-space has been proposed in order to deal with negative diffusion and has been shown to give good results in some non linear quantum systems. ${ }^{19,20}$

Here we consider the approximated method discussed in ${ }^{8,21}$ consisting in neglecting pump trajectories exceeding twice the threshold value. The approximation has been successfully used in a DOPO without PC for 
$A_{0}^{s t} \leq 1.5 A_{0}^{t h r}$ as an extremely large fluctuation in a trajectory would be necessary in order to lose the positiveness of the diffusion. Here we will concentrate in the region below and at threshold, where this approximation is well-justified.

With the inclusion of the new spatial dependent detunings and introducing the scaling

$$
\begin{aligned}
& \gamma_{0}=\gamma_{1}=\gamma, \quad a_{0}=a_{1} / 2=a, t^{\prime}=\gamma t, \quad \vec{x}^{\prime}=\frac{\vec{x}}{\sqrt{a}} \\
& A_{i}^{\prime}=\frac{g}{\gamma} A_{i}, \quad E^{\prime}=\frac{g}{\gamma^{2}} E, \quad \epsilon_{i}^{\prime}=\frac{g}{\gamma^{3 / 2} a^{D / 4}} \epsilon_{i}
\end{aligned}
$$

where $\mathrm{D}$ is the transversal dimensionality of the system, we obtain the equations

$$
\begin{aligned}
& \partial_{t} \alpha_{0}(\vec{x}, t)=-\left[\left(1+i \Delta_{0}(\vec{x})\right)-i \nabla^{2}\right] \alpha_{0}(\vec{x}, t)+E-\frac{1}{2} \alpha_{1}^{2}(\vec{x}, t)+\sqrt{\frac{2}{a}} \frac{g}{\gamma} \xi_{0}(\vec{x}, t) \\
& \partial_{t} \alpha_{1}(\vec{x}, t)=-\left[\left(1+i \Delta_{1}(\vec{x})\right)-2 i \nabla^{2}\right] \alpha_{1}(\vec{x}, t)+\alpha_{0}(\vec{x}, t) \alpha_{1}^{*}(\vec{x}, t)+\sqrt{\frac{2}{a}} \frac{g}{\gamma} \xi_{1}(\vec{x}, t) .
\end{aligned}
$$

with non-vanishing moments of the white noise:

$$
\left\langle\xi_{i}(\vec{x}, t) \xi_{j}^{*}\left(\vec{x}^{\prime}, t^{\prime}\right)\right\rangle=\delta_{i j} \delta\left(\vec{x}-\vec{x}^{\prime}\right)\left(t-t^{\prime}\right), \quad\left\langle\xi_{1}(\vec{x}, t) \xi_{1}\left(\vec{x}^{\prime}, t^{\prime}\right)\right\rangle=f\left[\alpha_{0}(\vec{x}, t)\right] \delta\left(\vec{x}-\vec{x}^{\prime}\right)\left(t-t^{\prime}\right) .
$$

We observe that the noise is phase sensitive and multiplicative, with moments of the signal noise depending on the value of the pump field. However due to the form of $\hat{H}$ (quadratic in $\hat{A}_{1}$ and linear in $\hat{A}_{0}$ ), these equations have the same formal expression in the Ito or Stratonovich interpretations. ${ }^{22}$ The condition (7) in the new variables is

$$
\left|\alpha_{0}(\vec{x}, t)\right|<2
$$

\section{INSTABILITY THRESHOLD}

The threshold for down-conversion in a DOPO is obtained from equations identical to (8) but neglecting the noise terms. In the case of homogeneous detunings

$$
\Delta_{0}(\vec{x})=\Delta_{0}, \quad \Delta_{1}(\vec{x})=\Delta_{1}
$$

these classical equations have a trivial homogeneous solution

$$
A_{1}^{s t}=0, A_{0}^{s t}=\frac{E}{1+i \Delta_{0}} .
$$

For $\Delta_{1}$ the zero homogeneous solution becomes unstable at $E=E_{t h r}=\sqrt{1+\Delta_{0}^{2}}$. The perturbations with maximum growth rate are those with wave number

$$
\left|k_{c}\right|=\sqrt{-\Delta_{1} / 2}
$$

and a pattern with this wave number is formed at threshold. ${ }^{23}$

It has been recently shown that the presence of an intracavity photonic crystal influences the instabilities in non linear cavities ${ }^{11,12}$ and is actually able to inhibit pattern formation. Here we consider one transverse dimension $(D=1)$ and a photonic crystal described by a sinusoidal modulation leading to detunings

$$
\begin{aligned}
& \Delta_{0}(x)=\Delta_{0}+I_{0} \sin k_{p} x \\
& \Delta_{1}(x)=\Delta_{1}+I_{1} \sin k_{p} x
\end{aligned}
$$

with $I_{i}$ amplitude of the modulation and $k_{p}$ transverse wavenumber of the the photonic crystal structure. Due to the modulation in the detunings, in general, there are not homogeneous stationary solutions of the classical 

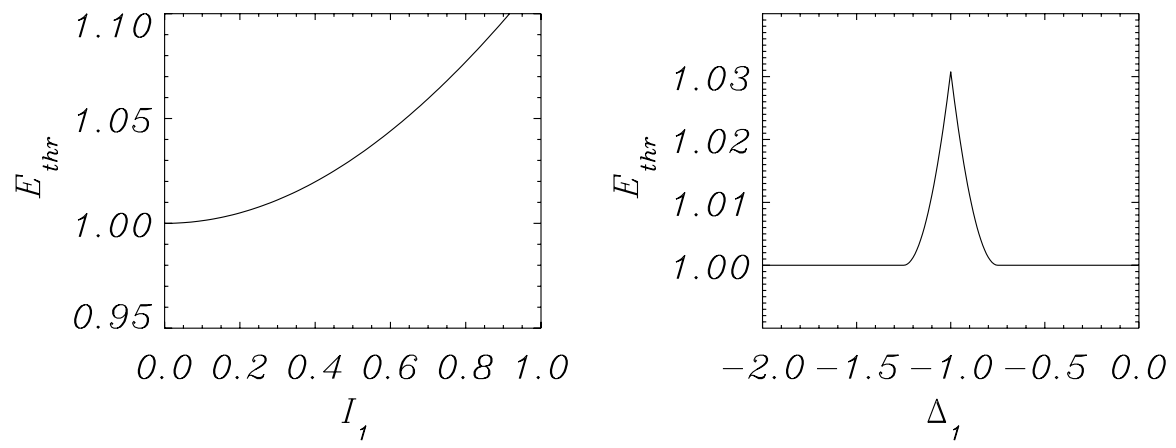

Figure 1. Instability threshold obtained with photonic crystals as in Eq. (14) for $\Delta_{0}=0, I_{0}=0, k_{p}=2 k_{c}$ and $\Delta_{1}=-1$ (left) and $I_{1}=0.5$ (right).

counterpart of Eqs. (8). In Fig. 1 we show the instability threshold obtained in the case of detuning modulation only in the signal field $\left(I_{0}=0\right)$. The stability analysis in this case is the same of a singly resonant DOPO, and the instability threshold has been analytically obtained by Gomila and Oppo. ${ }^{12}$ The DOPO threshold in this case is always increased with respect to the case without photonic crystal, whose value would be $E_{t h r}=1$. The photonic crystal has, therefore, the effect of inhibiting the instability. Inhibition was also observed in the case of a cubic instead of a quadratic non linearity, ${ }^{11}$ suggesting that this is a general effect of photonic crystals. We find, however, that this is not the case. The analysis of the DOPO's in which the modulation appears in the pump detuning instead of the signal, or in both fields, shows a more complex scenario. The threshold, for some parameters values, is found to be lowered by the presence of the photonic crystal, instead of being raised. With respect to what shown in Fig. 1 the threshold is found to be a non monotonic function of the modulation intensities $I_{i}$. As an example, for modulation frequency of the detuning at twice the critical one, $k_{p}=2 k_{c}$, numerically evaluated thresholds for $I_{0}=I_{1}=0.5$ is found at $E_{t h} \simeq 0.96$ and for $I_{0}=I_{1}=1.0$ is raised to $E_{t h} \simeq 1.03$.

In Fig. 2 we present results obtained by numerical simulation of the Langevin Eqs. (8). The integration method is described in previous papers. ${ }^{8,10,21}$ We show the average far field intensities for the pump and the signal light fields, just below threshold. In particular, instabilities occur just above the values $E=0.95,1.02$, and
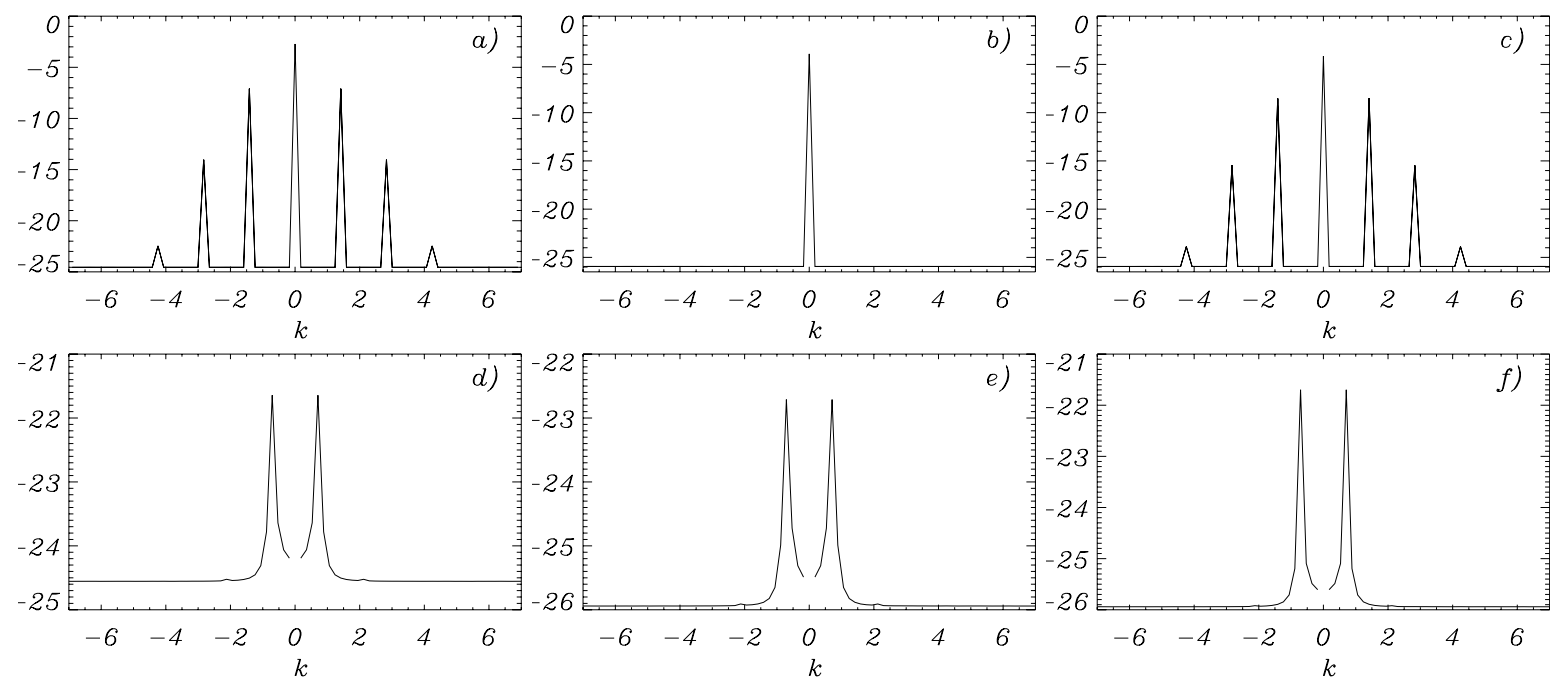

Figure 2. Logarithm of the pump (a,b,c) and signal (d,e,f) far field. Parameters: $\Delta_{0}=0, \Delta_{1}=-1$ and $k_{p}=2 k_{c}$. Three different cases, all of them below the respective thresholds: for $I_{0}=I_{1}=0.5$ with $E=0.95$, for $I_{0}=0.0$ and $I_{1}=0.5$ with $E=1.02$, for $I_{0}=0.5$ and $I_{1}=0.0$ with $E=0.93$. 
0.93 when detuning modulations appear in both fields, or only in the signal, or only in the pump, respectively. Even if these graphics do not correspond to what would be found by simulation of the classical (deterministic) counterpart of Eqs. (8), they still show that the macroscopic pump state is homogeneous only in the second case, for vanishing detuning modulation. In general several harmonics are excited in the pump field and this has clearly a relation with the threshold lowering in the presented cases.

\section{QUANTUM FLUCTUATIONS AND TWIN BEAMS CORRELATIONS}

Numerical simulations of Eqs. (8) allow to characterize the spatio-temporal dynamics of the quantum light fields and to calculate their possible quantum correlations. In particular, we focus here on the spatial distribution of quantum fluctuations (Fig. 2) and on twin beams correlations between opposite spatial modes. A previous work showed that these quantum correlations where present not only as quantum images, below threshold, but also in presence of an intense signal field, above threshold. ${ }^{8}$

Here we discuss the effect of the photonic crystal on quantum fluctuations both in the pump and in the signal light states. Non-classical features in the twin beams correlations are displayed for negative values of the normal-ordered variance of the difference of the two intensities ${ }^{8}$

$$
V(\vec{k})=\frac{\left\langle:\left[\hat{N}_{i}(\vec{k})-\hat{N}_{i}(-\vec{k})\right]^{2}:\right\rangle}{S . N \cdot\left(\hat{N}_{i}\right)} .
$$

normalized to the corresponding shot noise value $S . N .(\hat{N})$ for each $\vec{k}$. This value is proportional to the average of the sum of the intensities of the two beams with wavevectors $\pm \vec{k}$. Negative values of $V(\vec{k})$ indicate subPoissonian statistics for the intensity difference of the two beams at $\pm \vec{k} \cdot{ }^{24}$ Even if both the variance and the shot noise are functions of $\vec{k}$, in some cases the normalized quantity (16) is uniform. In a linear analytical treatment below threshold, for homogeneous pump and detunings, $V(k)=-0.5$, independently of the pump intensity and of the wave-vector. ${ }^{4,24}$ In the following we study how this variance is modified by the presence of the photonic crystal. As our stochastic equations provide average of antinormal ordered quantities, we present the relations between the antinormal and normal ordered expression of interest here.

\subsection{From antinormal to normal ordering}

The operators averages involved in this analysis are calculated in antinormal order with the Q representation and can be related to normal order expected values by resting the proper quantities. We consider, as an example, the normal ordered variance of the number operator $\hat{N}(\vec{k})=\hat{a}^{\dagger}(\vec{k}) \hat{a}(\vec{k})$

$$
: \triangle^{2} \hat{N}:=<: \hat{N}^{2}:>-<: \hat{N}:>^{2}=<\hat{a}^{\dagger}(\vec{k}) \hat{a}^{\dagger}(\vec{k}) \hat{a}(\vec{k}) \hat{a}(\vec{k})>-<\hat{a}^{\dagger}(\vec{k}) \hat{a}(\vec{k})>^{2}
$$

where we omit the index $i$, being the formulation equivalent for pump and signal fields operators. The commutator equals:

$$
C_{k k^{\prime}}=\left[\hat{a}_{i}(\vec{k}), \hat{a}_{i}^{\dagger}\left(\vec{k}^{\prime}\right)\right]=c \delta\left(\vec{k}-\vec{k}^{\prime}\right) .
$$

where $c$ is equal to one, before of field scalings (8). Normal (: :) and antinormal (: $:)$ ordered quantities are related by:

$$
\begin{array}{r}
: \hat{N}_{i}(\vec{k}): \equiv \hat{N}_{i}(\vec{k})=\vdots \hat{N}_{i}(\vec{k}) \vdots-C_{k k} \\
: \triangle^{2} \hat{N}:=\vdots \triangle^{2} \hat{N}:-2<\vdots \hat{N} \vdots>C_{k k}+C_{k k}^{2} .
\end{array}
$$

The shot noise is:

$$
S . N(\hat{N})=<\vdots \hat{N} \vdots>C_{k k}-C_{k k}^{2} .
$$

These relations are used to get normal ordered averages, like (16), from the antinormal ones obtained by numerical simulation of the stochastic spatio-temporal field dynamics in the $Q$ representation. Notice that discretization in space due to numerical simulation needs to be taken into account in order to properly evaluate the commutator. Numerical results can indeed be compared with analytical expression when possible, as for the twin beams correlations in a DOPO below threshold, without photonic crystal. ${ }^{4}$ 

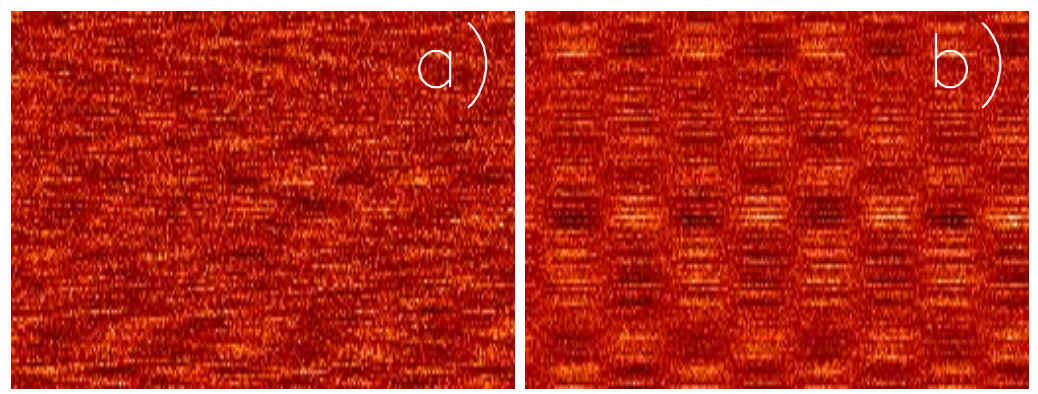

Figure 3. Near field quantum fluctuations in the real part of the signal field for parameters: a) $I_{0}=0, I_{1}=0.5, k_{p}=2 k_{c}$ and $E=1.02$ like in Fig. $2 \mathrm{~b}$ and e; and b) $I_{0}=0.5, I_{1}=0, k_{p}=2 k_{c}$ and $E=0.93$ like in Fig. 2 , c and $\mathrm{f}$.

\subsection{Numerical results}

We consider Eqs. (8) with phase sensitive multiplicative noise $\xi_{1}(x, t)$ fulfilling $(9)$ and numerically generated considering 8

$$
\xi_{1}(x, t)=\left[\frac{-\alpha_{0 I}(x, t)}{2 \sqrt{2+\alpha_{0 R}(x, t)}}+\frac{i}{2} \sqrt{2+\alpha_{0 R}(x, t)}\right] \phi(x, t)+\sqrt{\frac{1-\frac{\left|\alpha_{0}(x, t)\right|^{2}}{4}}{2+\alpha_{0 R}(x, t)}} \psi(x, t)
$$

with $\alpha_{0}=\alpha_{0 R}+i \alpha_{0 I}$ and $\phi, \psi$ uncorrelated real white noises in space and time, with variances one.

As mentioned in the previous Section, in Fig. 2 we show the average far field intensities for the pump and the signal light fields, just below threshold, in three different cases. Pump modulation in this regime appears only when the pump detuning is modulated and, in this case, the even spatial harmonics of $k_{c}$ are excited. Notice that, in absence of photonic crystals, these spatial modes would be intense only above threshold. The modulation of the photonic crystal has the wave-number of the spatial pattern that would arise above threshold, for an homogeneous DOPO, $k_{p}=2 k_{c}$. Other cases, $k_{p}=n k_{c}$, are under investigation.

The average signal field vanishes because we consider pump values $E$ just below threshold. As Fig. 2 d,e,f show, however, modes with the critical wave-number $\left(k=k_{c} \simeq 0.7\right.$ for our choice of parameters) are weakly damped and have a non vanishing average intensity. This is the regime of quantum images, extensively studied in the last decade and characterized by the "spatial structures manifested by the correlation functions" between the field at different points, and also by "noisy images" of the spatial fluctuations. ${ }^{25}$ Such noisy images in the near field do generally display spatial diffusion, arising from the translational invariance of the system in the transverse plane, as shown in Fig. 3a. This scenario can be modified in presence of a photonic crystal, when the stationary state below threshold is not homogeneous anymore. This happens, for instance, in the case displayed in Fig. 3b, where two modes with opposite phases appear weakly damped, and phase diffusion is completely suppressed. Breaking of the translational symmetry is a fundamental property of the new modulated system with respect to planar DOPO without photonic crystals. As numerical analysis above threshold also displays, the spatial phase of the solution fields, i.e. the position of the pattern in the DOPO, are indeed fixed and locked to the photonic crystal position.

In the following, we consider twin beams correlations below threshold, where it is well known the analytical expression for the intensity correlations without photonic crystal, within linear approximation for the fluctuations dynamics. ${ }^{4,8}$ In particular, the normalized variance (16) is homogeneous in $\vec{k}$ and is equal to -0.5 (black line in Fig. 4), as mentioned before. On the other hand, non-quantum twin beams correlations appear in the pump. In particular, the average intensity of opposite modes $\pm \vec{k}$ is the same (see symmetric plots in Fig. 2a,b,c), but the normal order variance vanishes $(V(\vec{k})=0)$.

In Fig. 4 we represent the normalized variance $V(k)$ corresponding to the signal field, for the three cases discussed above, whose mean intensities are represented in Fig. 2d,e,f. The signal correlations are still quantum and around -0.5 for most of the $k$ values but they tend to deteriorate around critical points, namely $k_{c}$ and $3 k_{c}$. This can be interpreted in the following way. In the considered case, the photonic crystal favors the generation 


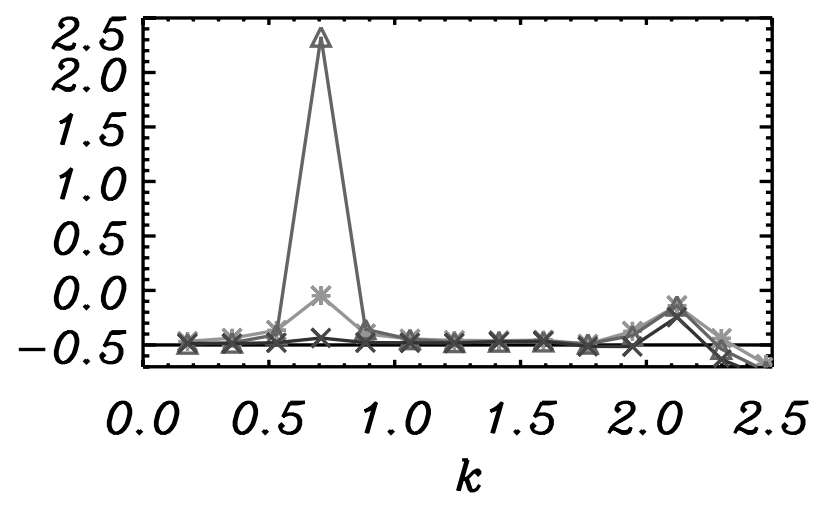

Figure 4. Normalized variance given by Eq.(16) in three different cases, like in Fig. 2 : for $I_{0}=I_{1}=0.5$ with $E=0.95$ (stars), for $I_{0}=0.0$ and $I_{1}=0.5$ with $E=1.02$ (triangles) and for $I_{0}=0.5$ and $I_{1}=0.0$ with $E=0.93$ (crosses). The analytical value without photonic crystal yields the black line represented at -0.5 . Quantum correlations correspond to negative values.

of spatial harmonics in the fields, even below threshold. The presence of these modes is associated to secondary processes like the ones discussed in a previous work, by some of us. ${ }^{6}$ These secondary processes give rise to the incoherent generation of photons with opposite wave-numbers and reduce the degree of correlation of photons generated in pairs. Interestingly, these correlations are quite robust crossing the instability threshold, and plots similar to Fig. 4 are found increasing the pump strength.

\section{CONCLUSIONS AND OUTLOOK}

We have shown some preliminary results on the quantum correlations in DOPO's with photonic crystals. Our main findings are that: (i) thresholds are raised or reduced by the photonic crystal depending on modulation strength; (ii) the average intensities of the fields are modified and spatial harmonics are excited; (iii) twin beams correlations persist both below and above threshold but can deteriorate in correspondence of some wave-vectors, due to the incoherent excitation of secondary processes. In general, we demonstrated that the detuning spatial modulation allows to tune the spatial profile of the intensity fluctuations.

New configurations, varying the spatial periodicity and strength of the photonic crystal, are under study, both from the classical (instability thresholds) and quantum (non-classical correlations) points of view. From one side, our objective is a general description of the control of instability threshold offered by photonic crystals. On the other side, we plan (i) to study the effect of the translational symmetry breaking in terms of the fluctuations of the transverse Poynting vector, or transverse momentum; and (ii) to characterize the effect of the photonic crystals on squeezing and EPR entanglement.

\section{ACKNOWLEDGMENTS}

We acknowledge financial support from the Spanish Government through project FISICOS (FIS2007-60327) and contract Ramon y Cajal, and from the Balearic Government, project 'Quantum light in microdevices' (PROGECIB-5A).

\section{REFERENCES}

[1] D.F. Walls and G.J. Milburn, Quantum Optics First Edition (Springer-Verlag, Berlin, 1994)

[2] M. I. Kolobov, "The spatial behavior of nonclassical light", Rev. Mod. Phys. 71, 1539 (1999)

[3] See Quantum Imaging, edited by M. I. Kolobov, (Springer, 2006) and references therein.

[4] A. Gatti, H. Wiedemann, L.A. Lugiato, I. Marzoli, G. L. Oppo, and S. M. Barnett, "Langevin treatment of quantum fluctuations and optical patterns in optical parametric oscillators below threshold", Phys. Rev. A 56, $877(1997)$ 
[5] L. A. Lugiato and F. Castelli, "Quantum noise reduction in a spatial dissipative structure", Phys. Rev. Lett. 68, 3284-3286 (1992)

[6] R. Zambrini and M. San Miguel, "Twin beams, non linearity and walk-off in Optical Parametric Oscillators", Phys. Rev. A 66, 023807 (2002)

[7] R.Zambrini, S.M.Barnett, P. Colet and M. San Miguel, "Macroscopic quantum fluctuations in noisesustained optical patterns", Phys. Rev. A 65, 023813 (2002).

[8] R. Zambrini, S.M. Barnett, P. Colet and M. San Miguel, "Non-classical behavior in multimode and disorderes transverse structures in OPO", Eur. Phys. J. D 22, 461-471 (2003)

[9] M. Hoyuelos, A. Sinatra, P. Colet, L. Lugiato and M. San Miguel, "Spatial pump-meter quantum correlations in a vectorial Kerr-medium model", Phys. Rev. A 59 1622-1632 (1999)

[10] R. Zambrini, M. Hoyuelos, A. Gatti, P. Colet, L. Lugiato, and M. San Miguel, "Quantum fluctuations in a continuous vectorial Kerr medium model " Phys. Rev. A 62, 63801 (2000).

[11] D. Gomila, R. Zambrini and G-L. Oppo, "Photonic Band-Gap Inhibition of Modulational Instabilities", Phys.Rev. Lett., 92 (2004)

[12] D. Gomila and G-L. Oppo, "Coupled-mode theory for photonic band-gap inhibition of spatial instabilities", Phys.R. E, 72, 016614 (2005)

[13] H. Carmichael, An Open Systems Approach to Quantum Optics, Lecture Notes in Physics, Vol. m18, (Springer, Berlin, 1993).

[14] S. M. Barnett, and P. M. Radmore, Methods in Theoretical Quantum Optics, (Oxford Science Publications, Oxford 1997).

[15] W. P. Schleich, Quantum Optics in Phase Space, (Wiley-VCH, Berlin, Germany, 2001).

[16] C. W. Gardiner and P. Zoller, Quantum Noise Second Edition (Springer-Verlag, Berlin, 2000)

[17] See U. Leonhardt, Measuring the Quantum State of Light, (Cambridge University Press, Cambridge, UK, 1997), Chapter VI and references therein.

[18] R.F. Pawula, "Approximation of the Linear Boltzmann Equation by the Fokker-Planck Equation", Phys.Rev. 162, 186 (1967).

[19] H.P. Yuen and P. Tombesi, "Langevin equations With negative diffusion coefficients. A new approach to quantum optics", Opt. Comm. 59, 155-159 (1986)

[20] R. Zambrini and S. M. Barnett, "Analytic stochastic treatment of a non linear quantum model with negative diffusion", Phys.Rev. A 65, 053810 (2002).

[21] M. Bache, P. Scotto, R. Zambrini, M. San Miguel and M. Saffman, "Quantum properties of transverse pattern formation in second-harmonic generation", Phys. Rev. A 66, 013809 (2002).

[22] C.W. Gardiner, Stochastic Methods, (Springer, Berlin, 1985)

[23] G-L. Oppo, M. Brambilla, and L. A. Lugiato, "Formation and evolution of roll patterns in optical parametric oscillators", Phys. Rev. A 49, 2028 (1994); G.-L. Oppo, M. Brambilla, D. Camesasca, A. Gatti, and L.A. Lugiato. "Spatiotemporal Dynamics of Optical Parametric Oscillators", J. Mod. Opt. 41, 1151 (1994).

[24] R. Zambrini and M. San Miguel, "Twin beams, non linearity, and walk-off in optical parametric oscillators", Phys. Rev. A 66, 023807 (2002).

[25] L. A. Lugiato, S. M. Barnett, A. Gatti, I. Marzoli, G. L. Oppo, and H. Wiedemann, pg. 5, in Coherence and Quantum Optics VII, edited by Eberly, Mandel, and Wolf, Plenum Press (New York, 1996). 